\section{RISK MANAGEMENT FOR SWIMSAFE - HELPING CHILDREN WITHOUT HARMING THEM}

${ }^{1}$ Michael Linnan, ${ }^{2}$ Stephen Beerman, ${ }^{3}$ Aminur Rahman, ${ }^{3}$ Fazlur Rahman, ${ }^{4}$ Justin Scarr. ${ }^{1}$ The Alliance for Safe Children; ${ }^{2}$ University of British Columbia, Canada; ${ }^{3}$ Centre for Injury Prevention and Research, Bangladesh; ${ }^{4}$ Royal Life Saving Society Australia

\subsection{6/injuryprev-2016-042156.827}

Background Child drowning in LMICs has become a major new public health issue. Drowning is the leading cause of death in children 1-17 yrs in Bangladesh. The lifecycle approach addresses child drowning for each age group. For children aged 4-14 years one primary intervention component involves teaching survival swimming using the SwimSafe curriculum. Risk management protocols have been developed to safely train children younger than 6 yrs old and older children with conditions that place them at risk.

Methods Community-based Participatory Research is ongoing in a rural community under injury surveillance. The partners in the research are UBC, CIPRB, TASC and RLSSA. SwimSafe is one of a suite of interventions. Children have been classified in 4 risk categories: Normal risk, increased risk, high risk and extreme risk. Different protocols that address each level of risk are being tested. Evidence of safety and training effectiveness is accumulating that will allow increased scale without increased risk to participating children.

Results Risk management is a very important for the SwimSafe intervention. About 1 out of 3 children under 6 yrs of age are in the increased risk category. For those 6 years and older, about 1 in $8-10$ are in the increased risk categories. Protocols are being tested that vary the instructor-child ratio, water depth, type of water body and presence of caretaker in the water with the child according to the level of increased risk for the child. Increased training for the swimming instructors, increased venue safety criteria, increased supervision and monitoring are being examined.

Conclusions Given the large proportion of the child population that is at increased risk when learning to swim, establishing risk management protocols that have evidence of safety is a priority for drowning prevention in Bangladesh and similar LMIC's. The goal is to safely teach these children basic swimming as most of them are at increased risk of drowning in their daily lives.

\section{NON-WEARING OF FLOTATION DEVICES AND SWIMMING ABILITY OF BOATING IMMERSION VICTIMS IN CANADA}

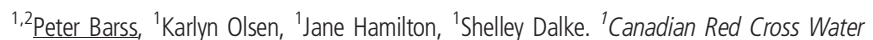
Safety Program; ${ }^{2}$ Division of Occupational and Environmental Health, School of Population and Public Health, University of British Columbia, Vancouver, Canada

\subsection{6/injuryprev-2016-042156.828}

Background Boating is the most frequent activity for waterrelated immersion deaths in Canada. Central in immersion/ drowning is non-wearing of personal flotation devices (PFDs). Anecdotal observation of frequent non-wearing among victims led to quantitive assessment using negative binomial and Poisson regression models.

Methods Annual Red Cross collection of 1991-2010 Canadian coroner data was by structured questionnaire. Analysis included ten variables in the final model.

Results There were 2678 recreational and daily living boating immersion deaths during 1991-2010. Certain variables significantly increased the odds of properly wearing a PFD and others decreased them. Controlling for all other variables, victims with average swimming ability had 1.93 times the odds of wearing compared with non and weak swimmers combined (95\% CI: 1.29-2.87). Strong swimmers had 1.90 times the odds of wearing compared with non/weak swimmers (95\% CI: 1.06-3.40).

Conclusion It was surprising that boating victims with low swimming ability were less likely than swimmers to have worn a flotation device. It is possible, but remains to be established, that poor swimmers were less likely to have participated in a swimming course, which included the main elements of water safety for boating and other activities.

\section{REVIEW OF RECREATIONAL AND OCCUPATIONAL PFD WEARING LEGISLATION AT PROVINCIAL, STATE, AND MUNICIPAL LEVELS IN CANADA AND INTERNATIONALLY}

${ }^{1}$ Shelley Dalke 1,2Peter Barss, 'Karlyn Olsen, ${ }^{1}$ Jane Hamilton. ${ }^{1}$ Canadian Red Cross Water Safety Program; ${ }^{2}$ Division of Occupational and Environmental Health, School of Population and Public Health, University of British Columbia, Vancouver, Canada

\subsection{6/injuryprev-2016-042156.829}

Background With a few notable exceptions, national legislation requiring wearing of a personal flotation device or lifejacket by boaters is uncommon. As a result some municipalities and provinces or states have introduced their own regulations.

Methods Information was obtained by searching of Safety Lit and other sources, including by contacting instigators of national legislation

Results Regulations for recreational and occupational boating were obtained for all 13 Canadian provinces and territories as well as one city. Canadian laws were diverse and are relatively weak, with the notable exception of the city of Calgary. Information was also obtained for Australia, Ireland, New Zealand, and the United States. In the United States, state laws supersede the national law, which appears mainly oriented towards children. The Irish legislation is more comprehensive than most. The most comprehensive state law in Australia is in Victoria State and has had remarkable success in improving outcomes as measured by boating drowning fatalities. In New Zealand, 18 regions and cities have regulations, some of which were comprehensive in requiring mandatory wearing. Many regulation featured opt out clauses at the discretion of the operator.

Conclusions Current legislation on wearing of flotation devices is variable. If the weak regulations in many jurisdictions were to be replaced by those such as in Victoria, Ireland and certain regions and cities in New Zealand, it is probable that many boating fatalities could be averted. Since children are uncommon victims of boating immersion in many high income countries, legislation targeting children will not be expected to be effective in such countries, with certain exceptions such as indigenous peoples. Rather the focus should be adult males

\section{DROWNING FROM THE VIEWPOINT OF THE NEAR- DROWNED}

Anneli Toivonen. Specialist for special swimming, the Finnish Swimming Teaching and Lifesaving Federation, Helsinki, Finland

\subsection{6/injuryprev-2016-042156.830}

Background The Finnish Swimming Teaching and Lifesaving Federation (FSL) is an educational and information-providing 